\title{
Análise Fatorial Confirmatória da Versão Portuguesa da Escala Multidimensional de Perfecionismo de Frost
}

\author{
Confirmatory Factor Analysis of the Portuguese Version of the Frost \\ Multidimensional Perfectionism Scale
}

\author{
Cláudia Carmo ${ }^{1}$, Marta Brás ${ }^{2}$, Luís Batista ${ }^{3}$ e Luís Faísca ${ }^{4}$
}

\begin{abstract}
Resumo
Este estudo teve como objetivo analisar as propriedades psicométricas e a dimensionalidade da versão portuguesa da Escala Multidimensional de Perfecionismo de Frost (FMPS) recorrendo à análise fatorial confirmatória. A amostra foi constituída por 832 estudantes do ensino secundário e universitário (65.0\% do sexo feminino), com uma média de idades de 19.5 anos. Os índices de ajustamento da análise fatorial confirmatória permitiram considerar o modelo multidimensional de seis fatores a melhor representação dos dados, replicando a solução original. Os resultados mostraram boas propriedades psicométricas. A consistência interna da escala apresentou coeficientes bastante satisfatórios (alfa de Cronbach=.853). O método teste-reteste sugeriu uma boa estabilidade temporal da escala $(r=.779)$. Globalmente a FMPS é um instrumento fiável e validado para o estudo do perfecionismo em Portugal.
\end{abstract}

Palavras-chave: Escala Multidimensional de Perfecionismo, análise fatorial confirmatória, português europeu

\begin{abstract}
This study aimed to analyse the psychometric properties and dimensionality of the Portuguese version of the Multidimensional Scale of Frost Perfectionism (FMPS) using the confirmatory factor analysis. The sample consisted of 832 high school and college students (65.0\% female) with a mean age of 19.5. The adjustment indexes of confirmatory factor analysis permitted to consider the multidimensional model of six factors the best representation of the data, replicating the original solution. The results showed good reliability and appropriate validity. The internal consistency of the scale presented satisfactory coefficients (Cronbach's alpha $=.853)$. The test-retest method suggested a good temporal stability of the scale $(r=.779)$. Globally the FMPS is a reliable and validated instrument for the study of perfectionism in Portugal.
\end{abstract}

Keywords: Frost Multidimensional Perfectionism Scale, factorial confirmatory analysis, european portuguese

\footnotetext{
${ }^{1}$ Doutorada em Psicologia pela Universidade do Algarve. Professora Auxiliar do Departamento de Psicologia e Ciências da Educação da Universidade do Algarve. Contactos: Faculdade de Ciências Humanas e Sociais. Campus de Gambelas, 8005 139 Faro, Portugal. Tel.: +351289800914. E-mail: cgcarmo@ualg.pt

${ }^{2}$ Doutorada em Psicologia pela Universidade do Algarve. Professora Auxiliar Convidada do Departamento de Psicologia e Ciências da Educação da Universidade do Algarve, Portugal. E-mail: mbras@ualg.pt

3 Mestre em Psicologia Clínica e da Saúde pela Universidade de Évora, Portugal. Psicólogo Clínico. E-mail: luisbatista87@gmail.com

${ }^{4}$ Doutorado em Psicologia pela Universidade do Algarve. Professor Auxiliar do Departamento de Psicologia e Ciências da Educação e investigador do CBMR - Centro de Investigação Biomédica da Universidade do Algarve, Portugal. E-mail: lfaisca@ualg.pt
} 


\section{Introdução}

Desde a última metade do século $\mathrm{XX}$ que o conceito de perfecionismo tem sido objeto de interesse generalizado para clínicos e investigadores no campo da psicologia (e.g., Adler, 1956; Hamachek, 1978; Hollender, 1965; Horney, 1950; Missildine, 1963; Pacht, 1984). Durante este período foram publicados mais de 1800 artigos científicos sobre o tema em revistas especializadas. O estudo do perfecionismo tem englobado áreas tão distintas como a natureza e dimensionalidade do construto (Hewitt, Flett, Sherry, \& McGee, 2003; Shafran, Cooper, \& Fairburn, 2003), aspetos cognitivos e metacognitivos do perfecionismo (Macedo et al., 2015), modelos explicativos do desenvolvimento e manutenção do perfecionismo (Flett, Hewitt, Oliver, \& Macdonald, 2002), perfecionismo infantil (Oros, 2003) e o seu papel na etiologia, manutenção e desenvolvimento de quadros psicopatológicos, incluindo perturbações do comportamento alimentar (Carvalho \& Novo, 2014), depressão e ansiedade (Gautreau, Sherry, Mushquash, \& Stewart, 2015), perturbação obsessivo-compulsiva (Pinto, Greene, Storch, \& Simpson, 2015), perturbações da personalidade (Egan, Wade, \& Shafran, 2011), perturbações do sono (Azevedo et al., 2010) e suicídio (O'Connor, 2007).

O perfecionismo tem sido definido como o "estabelecimento de padrões de desempenho excessivamente elevados, acompanhado de uma autoavaliação demasiado crítica" (Frost, Marten, Lahart, \& Rosenblate, 1990), estando esses padrões elevados associados ao medo de falhar (Flett, Blankstein, Hewitt, \& Koledin, 1992).

A grande variedade de contextos em que o perfecionismo tem sido estudado e a ausência de uma definição consensual desencadearam diversas perspetivas e abordagens deste construto.

Uma das controvérsias que divide teóricos, clínicos e investigadores diz respeito à sua dimensionalidade. Alguns autores conceptualizam o perfecionismo como um construto unidimensional (e.g., Shafran, Cooper, \& Fairburn, 2002), centrando-se nos aspetos intrapessoais; outros defendem uma perspetiva multidimensional (e.g., Frost et al., 1990; Hewitt et al., 2003) que integra não só dimensões intrapessoais, auto-orientadas, como também componentes interpessoais.

O aparecimento das conceções multidimensionais do perfecionismo no início dos anos 90, levaram Frost et al. (1990) a desenvolver a primeira medida de autorrelato para avaliar o perfecionismo segundo esta conceção, a Escala Multidimensional de Perfecionismo (FMPS Multidimensional Perfectionism Scale), reconhecida como uma das medidas de perfecionismo mundialmente mais utilizadas. A FMPS inclui dimensões intrapessoais, que avaliam o perfecionismo direcionado para o próprio, como os Padrões Pessoais (PP) excessivamente elevados, a Preocupação Excessiva com os Erros (PE), as Dúvidas sobre as Ações Pessoais (DA), a importância exagerada atribuída à precisão, à ordem e à Organização $(\mathrm{O})$; e dimensões interpessoais e desenvolvimentais, que refletem a perceção de exigências, nomeadamente as Expetativas Parentais (EP) e as Críticas Parentais (CP). Podem ainda ser mencionadas outras medidas de avaliação do perfecionismo: a Escala Multidimensional de Perfecionismo (HMPS - Multidimensional Perfectionism Scale; Hewitt \& Flett, 1991), e a Escala Quase Perfeita - Revista (APS-R - Almost Perfect Scale-Revised; Slaney, Rice, Mobley, Trippi, \& Ashby, 2001).

Estudos posteriores ao trabalho pioneiro de Frost et al. (1990) permitiram validar este instrumento em diferentes amostras, contextos e culturas. A FMPS foi traduzida e adaptada da língua inglesa para vários idiomas, como o castelhano (Gelabert et al., 2011), alemão (Altstotter-Gleich \& Bergemann, 2006), francês (Rheaume, Freeston, Dugas, Letarte, \& Ladouceur, 1995), chinês (Cheng, Chong, \& Wong, 1999; Zi \& Zhou, 2004), coreano (Lee \& Park, 2011), turco (Kagan, 2011) e português (Amaral et al., 2013) demonstrando em geral propriedades psicométricas adequadas.

A estrutura fatorial original de seis fatores foi replicada nalguns estudos (Altstotter-Gleich \& Bergemann, 2006; Amaral et al., 2013; Gelabert et al., 2011; Kagan, 2011; Parker \& Adkins, 1995; Parker \& Stumpf, 1995; Rheaume et al., 1995); contudo, nem sempre as evidências empíricas permitiram suportar essa estrutura. Em alternativa, 
outras soluções têm sido propostas por autores que sugerem estruturas de cinco fatores (Chan, 2009; Cheng et al., 1999; Cox, Enns, \& Clara, 2002; Lee \& Park, 2011; Paredes, Mancilla-Diaz, Rayon, Arevalo, \& Aguilar, 2010; Stallman \& Hurst, 2011; Zi \& Zhou, 2004), de quatro fatores (Harvey, Pallant, \& Harvey, 2004; Hawkins, Watt, \& Sinclair, 2006; Stoeber, 1998; Stumpf \& Parker, 2000) ou mesmo de três fatores (Purdon, Antony, \& Swinson, 1999); ver Quadro 1. Na grande maioria dos estudos de adaptação da FMPS, independentemente da solução fatorial adotada, recorre-se quase sempre à exclusão de alguns dos itens que compõe a escala original, oscilando entre um (Parker \& Adkins, 1995) e vinte (Chan, 2009) o número de itens eliminados, dando assim origem a versões reduzidas da escala.

O número de fatores que podem ser extraídos a partir das respostas aos 35 itens da FMPS é uma questão que ainda não reúne consenso. Contudo, é possível delinear padrões nas diferentes soluções apresentadas: as soluções de cinco fatores optam pela fusão do fator Preocupação com os Erros (PE) com o fator Dúvidas sobre as Ações (DA) (e.g., Cox et al., 2002); pela junção dos dois fatores de natureza parental, Expetativas Parentais (EP) e Críticas Parentais (CP) (e.g., Lee \& Park, 2011) ou pela extinção do fator $C P$, permanecendo apenas o fator EP (e.g., Paredes et al., 2010). As soluções de quatro fatores resultam de agrupar, agora em simultâneo, os fatores EP e $\mathrm{CP}$ e os fatores PE e DA (e.g., Hawkins et al., 2006). Por último, a versão com três fatores proposta para a FMPS, apresentada por Purdon et al. (1999), mantém as fusões presentes nas soluções de quatro fatores e acrescenta uma nova união entre o fator Padrões Pessoais (PP) e o fator Organização (O), sugerindo as seguintes renomeações: Medo de Errar (PE/DA), Pressão Parental Percebida (EP/CP) e Orientação para Objetivos de Realização (PP/O).

A diversidade das soluções fatoriais propostas nos estudos de adaptação da Escala Multidimensional de Perfecionismo a diferentes línguas e culturas sublinha a relevância do contexto sociocultural na avaliação do perfecionismo. Neste âmbito, e sendo a FMPS um dos instrumentos com maior tradição empírica e influência no estudo do perfecionismo, considerou-se pertinente analisar a Revista Iberoamericana de Diagnóstico y Evaluación - e Avaliação Psicológica. RIDEP · Nº44 · Vol.2 · 28-43 2017 dimensionalidade da versão portuguesa desta escala. $\mathrm{O}$ estudo anteriormente conduzido com a FMPS em Português Europeu (Amaral et al., 2013) considerou que o modelo original de seis fatores seria adequado, embora os dados fossem também compatíveis com uma solução de menor dimensionalidade. Estes autores utilizaram a técnica da análise fatorial exploratória numa amostra de estudantes universitários maioritariamente feminina.

O presente estudo teve assim como principal objetivo analisar as propriedades psicométricas e a dimensionalidade da versão portuguesa da FMPS numa perspetiva confirmatória, recorrendo a uma amostra alargada de jovens e jovens adultos de ambos os sexos. Como objetivos específicos pretende-se (1) analisar a estrutura interna da FMPS, avaliando a possibilidade de replicar na amostra em estudo a estrutura fatorial encontrada pelos autores da versão original; (3) avaliar a fiabilidade das medidas da FMPS; (4) analisar a validade convergente e discriminante da FMPS; (5) analisar a validade concorrente da FMPS e, por último; (5) identificar diferenças entre grupos (sexo e escolaridade).

\section{Método}

\section{Participantes}

A amostra foi composta por 832 estudantes, 541 do sexo feminino (65.0\%) e 291 do sexo masculino (35.0\%), com idades compreendidas entre os 16 e os 30 anos $(M=19.48, D P=2.47)$. Mais de metade da amostra frequentava o ensino superior $(55.3 \%)$ e $44.7 \%$ frequentava o ensino secundário, tendo a amostra uma média de 12.74 $(D P=1.49)$ anos de escolaridade.

Uma subamostra de 119 participantes (14.3\% da amostra total) preencheu novamente os questionários, após um intervalo de tempo que variou entre as quatro e as cinco semanas. A comparação entre a subamostra $(N=119)$ e a amostra total $(N=832)$ não evidenciou diferenças estatisticamente significativas - idade $\left(t_{(118)}=-.66\right.$, $p=.511)$, sexo $\left(\chi_{(1)}^{2}=1.62, p=.203\right)$, estado civil $\left(\chi_{(1)}^{2}=.05, p=.827\right)$, nível de ensino $\left(\chi_{(1)}^{2}=.06\right.$, $p=.810)$-, pelo que poderemos considerar que a amostra de reteste não se distingue da amostra 
Quadro 1. Síntese dos Estudos da Estrutura Fatorial da FMPS: Autoria, Ano, Idioma e País da Versão, Amostra Estudada, Método de Análise Fatorial e Dimensões Identificadas

\begin{tabular}{|c|c|c|c|c|}
\hline Estudo & Língua/ País & Amostra & Método & Dimensões \\
\hline $\begin{array}{l}\text { Frost, Marten, Lahart e } \\
\text { Rosenblate (1990) }\end{array}$ & Inglês/EUA & $\begin{array}{c}N=232(100 \% \text { sexo } \\
\text { feminino }) \\
\text { Estudantes universitários }\end{array}$ & AFE & $\begin{array}{c}6 \text { fatores (35 itens) } \\
\text { PE, PP, EP, CP, DA, O }\end{array}$ \\
\hline Parker e Adkins (1995) & Inglês/EUA & $\begin{array}{c}N=278(61.5 \% \text { sexo } \\
\text { feminino }) \\
\text { Estudantes universitários }\end{array}$ & AFE & $\begin{array}{c}6 \text { fatores (34 itens) } \\
\text { PE, PP, EP, CP, DA, O }\end{array}$ \\
\hline $\begin{array}{l}\text { Parker e Stumpf } \\
\text { (1995) }\end{array}$ & Inglês/EUA & $\begin{array}{c}N=855(38.0 \% \text { sexo } \\
\text { feminino }) \\
\text { Estudantes ensino } \\
\text { secundário, sobredotados }\end{array}$ & $\mathrm{AFC}$ & $\begin{array}{c}6 \text { fatores }(35 \text { itens }) \\
\text { PE, PP, EP, CP, DA, O }\end{array}$ \\
\hline $\begin{array}{l}\text { Rheaume, Freeston, } \\
\text { Dugas, Letarte e } \\
\text { Ladouceur (1995) }\end{array}$ & $\begin{array}{l}\text { Francês/ } \\
\text { Canadá }\end{array}$ & $\begin{array}{c}\quad N=245(83.7 \% \text { sexo } \\
\text { feminino }) \\
\text { Estudantes universitários }\end{array}$ & AFE & $\begin{array}{c}6 \text { fatores }(31 \text { itens }) \\
\text { PE, PP, EP, CP, DA, O }\end{array}$ \\
\hline $\begin{array}{l}\text { Altstotter-Gleich e } \\
\text { Bergemann }(2006)^{(a)}\end{array}$ & $\begin{array}{l}\text { Alemão/ } \\
\text { Alemanha }\end{array}$ & $\begin{array}{c}N=1170(60.0 \% \text { sexo } \\
\text { feminino }) \\
\text { Estudantes universitários }\end{array}$ & $\mathrm{AFC}$ & $\begin{array}{c}6 \text { fatores }^{(\mathrm{a})} \\
\mathrm{PE}, \mathrm{PP}, \mathrm{EP}, \mathrm{CP}, \mathrm{DA}, \mathrm{O}\end{array}$ \\
\hline $\operatorname{Kagan}(2011)^{(a)}$ & $\begin{array}{l}\text { Turco/ } \\
\text { Turquia }\end{array}$ & $\begin{array}{c}N=492(62.8 \% \text { sexo } \\
\text { feminino }) \\
\text { Estudantes universitários }\end{array}$ & $\mathrm{AFC}$ & $\begin{array}{c}6 \text { fatores }^{(b)} \\
\text { PE, PP, EP, CP, DA, O }\end{array}$ \\
\hline Gelabert et al. (2011) & $\begin{array}{l}\text { Castelhano/ } \\
\text { Espanha }\end{array}$ & $\begin{array}{c}N=582(100 \% \text { sexo } \\
\text { feminino }) \\
\text { Estudantes universitárias }\end{array}$ & $\mathrm{AFC}$ & $\begin{array}{c}6 \text { fatores }(35 \text { itens }) \\
\text { PE, PP, EP, CP, DA, O }\end{array}$ \\
\hline Amaral et al. (2013) & $\begin{array}{l}\text { Português/ } \\
\text { Portugal }\end{array}$ & $\begin{array}{c}N=217(82.0 \% \text { sexo } \\
\text { feminino }) \\
\text { Estudantes universitárias }\end{array}$ & $\mathrm{AFC}$ & $\begin{array}{c}6 \text { fatores }^{(\mathrm{c})}(35 \text { itens }) \\
\text { PE, PP, EP, CP, DA, O }\end{array}$ \\
\hline $\begin{array}{l}\text { Cheng, Chong e Wong } \\
\text { (1999) }\end{array}$ & $\begin{array}{l}\text { Chinês/ } \\
\text { China }\end{array}$ & $\begin{array}{c}N=947(59.0 \% \text { sexo } \\
\text { feminino }) \\
\text { Estudantes ensino } \\
\text { secundário }\end{array}$ & AFE & $\begin{array}{l}5 \text { fatores (27 itens) } \\
\text { PE, PP, EP, DA, O }\end{array}$ \\
\hline $\begin{array}{l}\text { Cox, Enns e Clara } \\
(2002)\end{array}$ & $\begin{array}{l}\text { Inglês/ } \\
\text { Canadá }\end{array}$ & $\begin{array}{l}N=288(63.2 \% \text { sexo } \\
\text { feminino }) \\
\text { Estudantes universitários }\end{array}$ & $\mathrm{AFC}$ & $\begin{array}{c}5 \text { fatores (22 itens) } \\
\text { PE, PP, EP/CP, DA, O }\end{array}$ \\
\hline Chan (2009) & $\begin{array}{l}\text { Chinês/ } \\
\text { China }\end{array}$ & $\begin{array}{c}\quad N=380(40.8 \% \text { sexo } \\
\text { feminino }) \\
\text { Estudantes ensino primário e } \\
\text { secundário, sobredotados }\end{array}$ & $\mathrm{AFC}$ & $\begin{array}{c}5 \text { fatores }(15 \text { itens }) \\
\text { PE/DA, PP, EP, CP, O }\end{array}$ \\
\hline $\begin{array}{l}\text { Paredes, Mancilla- } \\
\text { Diaz, Rayon, Arevalo } \\
\text { e Aguilar (2010) }\end{array}$ & $\begin{array}{l}\text { Castelhano/ } \\
\text { México }\end{array}$ & $\begin{array}{c}N=862(54.1 \% \text { sexo } \\
\text { feminino }) \\
\text { Estudantes ensino } \\
\text { preparatório e secundário }\end{array}$ & AFE & $\begin{array}{l}5 \text { fatores (29 itens) } \\
\text { PE, PP, EP, DA, O }\end{array}$ \\
\hline Lee e Park (2011) & $\begin{array}{l}\text { Coreano/ } \\
\text { C. do Sul }\end{array}$ & $\begin{array}{c}N=213(46.9 \% \text { sexo } \\
\text { feminino }) \\
\text { Estudantes universitários }\end{array}$ & AFC & $\begin{array}{c}5 \text { fatores (26 itens) } \\
\text { PE, PP, EP/CP, DA, O }\end{array}$ \\
\hline $\begin{array}{l}\text { Stallman e Hurst } \\
\text { (2011) }\end{array}$ & $\begin{array}{l}\text { Australiano/ } \\
\text { Austrália }\end{array}$ & $\begin{array}{c}N=6449(64.6 \% \text { sexo } \\
\text { feminino }) \\
\text { Estudantes universitários }\end{array}$ & $\begin{array}{l}\mathrm{AFE} \\
\mathrm{AFC}\end{array}$ & $\begin{array}{c}5 \text { fatores (29 itens) } \\
\text { PE, PP, EP/CP, DA, O }\end{array}$ \\
\hline Zi e Zhou (2004) $)^{(a)}$ & $\begin{array}{l}\text { Chinês/ } \\
\text { China }\end{array}$ & & (b) & $\begin{array}{l}5 \text { fatores ( } 27 \text { itens) } \\
\text { PE, PP, EP, DA, O }\end{array}$ \\
\hline Stoeber (1998) & $\begin{array}{l}\text { Alemão/ } \\
\text { Alemanha }\end{array}$ & $\begin{array}{c}N=243(66.2 \% \text { sexo } \\
\text { feminino }) \\
\text { Estudantes universitários }\end{array}$ & AFE & $\begin{array}{c}4 \text { fatores (33 itens) } \\
\text { PE/DA, PP, EP/CP, O }\end{array}$ \\
\hline $\begin{array}{l}\text { Stumpf e Parker } \\
(2000)\end{array}$ & Inglês/EUA & $\begin{array}{c}N=855(38.0 \% \text { sexo } \\
\text { feminino }) \\
\text { Estudantes ensino }\end{array}$ & AFE & $\begin{array}{c}4 \text { fatores (32 itens) } \\
\text { PE/DA, PP, EP/CP, O }\end{array}$ \\
\hline
\end{tabular}




\begin{tabular}{|c|c|c|c|c|}
\hline & & secundário, sobredotados & & \\
\hline $\begin{array}{l}\text { Harvey, Pallant e } \\
\text { Harvey (2004) }\end{array}$ & $\begin{array}{l}\text { Inglês/ } \\
\text { Austrália }\end{array}$ & $\begin{array}{c}N=255(55.7 \% \text { sexo } \\
\text { feminino }) \\
\text { Adultos }\end{array}$ & AFE & $\begin{array}{c}4 \text { fatores (35 itens) } \\
\text { PE/DA, PP, EP/CP, O }\end{array}$ \\
\hline $\begin{array}{l}\text { Khawaja e Armstrong } \\
(2005)\end{array}$ & $\begin{array}{l}\text { Inglês/ } \\
\text { Austrália }\end{array}$ & $\begin{array}{c}N=271(75.0 \% \text { sexo } \\
\text { feminino }) \\
\text { Estudantes universitários }\end{array}$ & AFE & $\begin{array}{c}4 \text { fatores (24 itens) } \\
\text { PE/DA, PP, EP/CP, O }\end{array}$ \\
\hline $\begin{array}{l}\text { Hawkins, Watt e } \\
\text { Sinclair (2006) }\end{array}$ & $\begin{array}{l}\text { Inglês/ } \\
\text { Austrália }\end{array}$ & $\begin{array}{c}N=409(100 \% \text { sexo } \\
\text { feminino }) \\
\text { Estudantes do ensino } \\
\text { secundário }\end{array}$ & AFC & $\begin{array}{c}4 \text { fatores (33 itens) } \\
\text { PE/DA, PP, EP/CP, O }\end{array}$ \\
\hline $\begin{array}{l}\text { Purdon, Antony e } \\
\text { Swinson (1999) }\end{array}$ & $\begin{array}{l}\text { Inglês/ } \\
\text { Canadá }\end{array}$ & $\begin{array}{c}N=322(49.7 \% \text { sexo } \\
\text { feminino }) \\
\text { Adultos (diagnosticados - } \\
\text { Perturbação de Ansiedade) }\end{array}$ & AFE & $\begin{array}{c}3 \text { fatores (30 itens) } \\
\text { PE/DA, PP/O, EP/CP }\end{array}$ \\
\hline
\end{tabular}

Nota. AFE=Análise Fatorial Exploratória; $\mathrm{AFC}=$ nálise Fatorial Confirmatória. PE=Preocupação com os Erros; $\mathrm{PP}=$ Padrões Pessoais; EP=Expetativas Parentais; $\mathrm{CP}=\mathrm{Críticas} \mathrm{Parentais;} \mathrm{DA=Dúvidas} \mathrm{sobre} \mathrm{as} \mathrm{Ações;} \mathrm{O}=\mathrm{Organização.}$

${ }^{a}$ Resumo disponível em língua inglesa; ${ }^{\mathrm{b}}$ Informação disponibilizada insuficiente; ${ }^{\mathrm{c}}$ Os autores consideraram uma outra solução alternativa de 4 fatores (PE/DA, PP, EP/CP, O).

original em termos da sua caracterização sociodemográfica.

\section{Medidas}

\section{A Escala Multidimensional de Perfecionismo de Frost}

Originalmente a FMPS (Frost et al., 1990) foi composta por um conjunto de 67 itens, baseados quer nas conceções teóricas do perfecionismo até então publicadas (e.g., Hamachek, 1978) quer em itens oriundos de medidas de perfecionismo já existentes, como a Escala de Perfecionismo de Burns (BPS - Burns Perfectionism Scale; Burns, 1980), a subescala Perfecionismo do Inventário de Perturbações Alimentares (EDI-P - Eating Disorder Inventory; Garner, Olmsted, \& Polivy, 1983) e uma medida de sintomatologia obsessivocompulsiva, o Inventário Obsessivo-Compulsivo de Maudsley (MOCI - Maudsley ObsessiveCompulsive Inventory; Rachman \& Hodgson, 1977). Numa amostra de 232 estudantes universitárias, Frost et al. analisaram a estrutura fatorial e a fiabilidade da primeira versão do instrumento, que conduziria a uma solução de seis fatores e à redução do número inicial de itens. Atualmente, a FMPS é composta por 35 itens, cada um deles avaliado numa escala de cinco pontos, onde 1 corresponde a Discordo completamente e 5 a Concordo completamente, correspondendo a valores elevados de concordância um maior nível de perfecionismo.

Os itens da FMPS agrupam-se em seis subescalas: Padrões Pessoais (PP), que avalia a tendência para estabelecer padrões pessoais excessivamente elevados que se tornam impossíveis de alcançar de forma satisfatória (e.g., "Eu tenho objetivos extremamente elevados"); Preocupação com os Erros (PE), que reflete as reações negativas perante a possibilidade de errar (e.g., "Eu devo ficar aborrecido se cometo um erro"); Dúvidas sobre as Ações (DA), que avalia a tendência para duvidar das suas capacidades para alcançar os objetivos propostos e para duvidar da qualidade das suas próprias ações (e.g., "Mesmo quando faço algo muito cuidadosamente, sinto frequentemente que não está muito bem”); Expetativas Parentais (EP), que denota a perceção de expetativas parentais extremamente elevadas (e.g., "Os meus pais queriam que eu fosse o melhor em tudo"); Críticas Parentais (CP), que reflete a perceção de que os pais eram/são excessivamente críticos (e.g., "Nunca senti que conseguia atingir os padrões dos meus pais"); e por último, a dimensão Organização (O), que reflete a importância atribuída à ordem e à precisão (e.g., "Procuro ser uma pessoa arrumada").

No estudo original de Frost et al. (1990), a pontuação total da escala, assim como as diferentes subescalas demonstraram uma adequada consistência interna, apresentando valores do coeficiente alfa de Cronbach entre .77 e .93. Mais especificamente, .83 (PP), .88 (PE), .77 (DA), .84 (EP), .84 (CP), .93 (O) e .90 para a pontuação total. Ainda neste estudo original, a validade concorrente da escala foi suportada, 
através de elevadas correlações da FMPS com outras medidas de perfecionismo, $r=.85 \mathrm{com}$ a Escala de Perfecionismo de Burns (BPS - Burns Perfectionism Scale; Burns, 1980) e $r=.59$ com a subescala Perfecionismo do Inventário de Distúrbios Alimentares (EDI-P - Eating Disorder Inventory; Garner, Olmsted, \& Polivy, 1983). A validade de construto da FMPS foi também suportada pela comparação com o Questionário de Experiências Depressivas (DEQ - Depressive Experiences Questionnaire; Blatt, D'Afflitti, \& Quinlan, 1976) $(r=.45$ para a dimensão Self Crítico e $r=.29$ para a dimensão Dependência). Num estudo posterior (Frost, Heimberg, Holt, Mattia, \& Neubauer, 1993), a FMPS apresentou também correlações significativas no sentido esperado com as dimensões da Escala Multidimensional do Perfecionismo de Hewitt e Flett (HMPS; 1991).

\section{A Escala Multidimensional de Perfecionismo de Hewitt}

A HMPS (Hewitt \& Flett, 1991) avalia três dimensões do perfecionismo: a dimensão Perfecionismo Auto-orientado (PAO), que avalia o estabelecimento de padrões excessivamente elevados e a motivação perfecionista dirigida a si próprio; o Perfecionismo Socialmente Prescrito (PSP), que avalia a perceção de que os outros estabelecem padrões excessivamente elevados dirigidos a si; e por último, a dimensão Perfecionismo Orientado para os Outros (POO), que avalia os padrões de desempenho ou de comportamento irrealistas para com os outros significativos. Assim, a principal distinção entre estas três dimensões diz respeito à origem e à direção do comportamento perfecionista.

A HMPS inclui 45 itens, avaliados numa escala de resposta com 7 níveis de concordância (entre 1 - Discordo completamente e 7 - Concordo completamente).

Os itens encontram-se distribuídos por três subescalas: Perfecionismo Auto-orientado (18 itens; e.g., "Preocupo-me em ter um resultado perfeito em tudo"); Perfecionismo Socialmente Prescrito (14 itens; e.g., "Sinto que as outras pessoas exigem demais de mim") e Perfecionismo Orientado para os Outros (8 itens; e.g., "Espero muito das pessoas que são importantes para mim")

Revista Iberoamericana de Diagnóstico y Evaluación - e Avaliação Psicológica. RIDEP · No44 · Vol.2 · 28-43 · 2017
A versão original apresenta uma boa consistência interna, demonstrando validade convergente e discriminante, bem como validade preditiva num amplo espetro de diagnósticos psiquiátricos (e.g., Egan et al., 2011).

A HMPS encontra-se traduzida e adaptada para a população portuguesa por Soares, Gomes, Macedo e Azevedo (2003) e apresenta qualidades psicométricas adequadas, sendo esta a versão utilizada neste estudo.

\section{Procedimentos}

Após a permissão por escrito do autor da FMPS iniciou-se o processo de tradução de acordo com os procedimentos sugeridos por van de Vijver e Hambleton (1996). Num primeiro momento, realizou-se a tradução da escala original, de inglês para português, sendo essa tradução posteriormente corrigida por dois juízes independentes. Num segundo momento, realizouse a retroversão independente por um psicólogo bilingue de língua materna inglesa que desconhecia a versão original. Por fim, foram confrontadas as três versões (original, tradução e retroversão) e, em contacto com os tradutores, efetuaram-se pequenas alterações na versão traduzida, de modo a preservar o sentido dos itens originais (Moreira, 2009). As instruções originais de preenchimento da escala também foram objeto de tradução e retroversão, mantendo-se o aspeto gráfico semelhante ao da escala americana.

A recolha de amostra foi realizada em escolas secundárias da região do Algarve e na Universidade do Algarve. A todos os alunos foi efetuado um pedido de participação voluntária, anónima e confidencial, sendo estes informados que se tratava da adaptação para a língua portuguesa de um instrumento de medida sobre a personalidade. Em seguida, foi dado a conhecer aos alunos que a sua participação consistia no preenchimento de questionários de autorresposta e foram lidas em voz alta as instruções gerais de preenchimento. Os alunos completaram o protocolo de avaliação de forma coletiva em contexto de sala de aula, na presença de um dos investigadores, sendo o tempo médio de preenchimento da FMPS cerca de 15 minutos.

A análise da estrutura fatorial da FMPS foi realizada numa perspetiva confirmatória, com 
recurso ao software AMOS versão 16.0 (Analysis of Moment Structures) que permite a modelização de dados através de equações estruturais. Para proceder à apreciação dos modelos propostos na literatura para a estrutura fatorial da FMPS, recorreu-se a diversos índices de qualidade do ajustamento: o teste de ajustamento do Quiquadrado e respetivos graus de liberdade; Raiz padronizada da média quadrática residual (Standardized Root Mean Square Residual, SRMSR), Índice de Tucker-Lewis (Tucker-Lewis Index, TLI), Índice de ajustamento comparativo (Comparative Fit Index, CFI), Índice de ajustamento normalizado de parcimónia (Parsimonious Normed Fit Index, PNFI), Raiz da média quadrática do erro de aproximação (Root Mean Square Error of Approximation, RMSEA) e Índice esperado de validação cruzada (Expected Cross-Validation Index, ECVI). Seguindo a proposta de Kline (2011), considerou-se que o ajustamento de um modelo aos dados é adequado para valores $\chi^{2} / g l$ entre 1 e 2; para valores de CFI, TLI igual ou superior a .9; para valores de RMSEA inferiores a .05; para valores de SRMSR inferiores a .08 , e para valores de PNFI maiores do que .6. Finalmente, ao comparar dois modelos, aquele com menor ECVI será o mais estável na população.

As restantes análises foram realizadas com recurso ao programa de tratamento de dados estatísticos Statistical Package for the Social Sciences (SPSS) (19.0) para Windows e ao programa Microsoft Office Excel 2010 (para calcular as magnitudes das diferenças através do $d$ de Cohen (1988)).

Quarenta e oito inquiridos (5.8\%) apresentavam valores omissos nas respostas à FMPS (oito inquiridos não responderam a dois itens e os restantes apenas omitiram uma resposta), perfazendo um total de 56 respostas omissas $-0.2 \%$ do total de respostas). Para impedir problemas inerentes à presença de valores omissos em análises de natureza correlacional, procedeu-se à sua estimação e imputação através do algoritmo EM (Expectation Maximization), utilizando as respostas aos restantes itens do questionário para estimar os valores em falta. Nenhum dos itens da FMPS apresentou indicadores extremos de assimetria (|coeficiente de assimetria| < 1.4) nem de curtose (|coeficiente Revista Iberoamericana de Diagnóstico y Evaluación - e Avaliação Psicológica. RIDEP · N44 · Vol.2 · 28-43 · 2017 de curtose $\mid<2.3$ ), sugerindo não estarmos perante violações severas à normalidade dos dados.

\section{Resultados}

\section{Análise fatorial confirmatória}

Foi analisado o modelo de seis fatores proposto originalmente por Frost et al. (1990) e, posteriormente testaram-se as várias soluções fatoriais alternativas identificadas na revisão de literatura (Quadro 1): modelo de cinco fatores com a fusão das dimensões relativas à perceção das expetativas parentais - EP e CP; modelo de cinco fatores com fusão das dimensões relativas às componentes intrapessoais negativas - $\mathrm{PE} e$ DA; modelo de quatro fatores, que reúne as duas fusões dos modelos de cinco fatores; e modelo de três fatores - que, para além das fusões já contempladas, reúne ainda o fator $\mathrm{PP}$ ao $\mathrm{O}$ (dimensões relativas às componentes intrapessoais adaptativas). A definição destes modelos foi sustentada na sua especificidade concetual, sem considerar os itens que foram eliminados de forma ad-hoc nos diferentes estudos. Desta forma, o número de fatores variou, mantendo-se sempre os 35 itens da versão original. Nenhum dos modelos testados é ortogonal. No Quadro 2 são apresentados os índices de ajustamento para os modelos testados.

Nenhum dos cinco modelos analisados mostra um ajustamento satisfatório. Como seria de esperar, atendendo à dimensão da amostra $(N=832)$, a estatística $\chi^{2}$ é significativa para todos os modelos testados $(p<.001)$. No entanto, o modelo com seis fatores (6F) e o segundo modelo com 5 fatores $(5 \mathrm{Fb})$ são aqueles que parecem proporcionar o melhor ajustamento. A comparação entre estes dois modelos revela que, apesar de uma maior complexidade, a melhoria no ajustamento demonstrada pelo modelo de $6 \mathrm{~F}$ é estatisticamente significativa $\left(\chi^{2}=255.8, \mathrm{gl}=5\right.$, $p<.001)$. No mesmo sentido vai o índice de parcimónia (PNFI), que sugere um melhor ajustamento do modelo $6 \mathrm{~F}$, não obstante a sua maior complexidade e o índice ECVI, que indica uma maior estabilidade. Desta forma, podemos afirmar que o modelo original de seis fatores de Frost et al. (1990) oferece melhor ajustamento aos dados da amostra portuguesa em estudo do que os 
Quadro 2. Índices de Ajustamento para os Modelos Testados

\begin{tabular}{|c|c|c|c|c|c|c|c|c|c|}
\hline Modelo & $\chi^{2}$ & $\mathrm{gl}$ & $\chi^{2} / g 1$ & SRMSR & TLI & CFI & PNFI & $\begin{array}{l}\text { RMSEA } \\
\text { (IC 95\%) }\end{array}$ & ECVI \\
\hline Modelo 6F & 2225.7 & 545 & 4.1 & .075 & .837 & .851 & .744 & $\begin{array}{c}.061 \\
(.058-.064)\end{array}$ & 2.88 \\
\hline $\begin{array}{l}\text { Modelo 5Fa } \\
\text { (EP/CP) }\end{array}$ & 2530.7 & 550 & 4.6 & .077 & .810 & .824 & .727 & $\begin{array}{c}.066 \\
(.063-.068)\end{array}$ & 3.24 \\
\hline $\begin{array}{l}\text { Modelo } 5 \mathrm{Fb} \\
\text { (PE/DA) }\end{array}$ & 2481.5 & 550 & 4.5 & .077 & .829 & .828 & .731 & $\begin{array}{c}.065 \\
(.062-.068)\end{array}$ & 3.18 \\
\hline $\begin{array}{l}\text { Modelo 4F } \\
\text { (PE/DA; } \\
\text { EP/CP) }\end{array}$ & 2751.4 & 554 & 5.0 & .078 & .790 & .805 & .715 & $\begin{array}{c}.069 \\
(.067-.072)\end{array}$ & 3.49 \\
\hline $\begin{array}{l}\text { Modelo 3F } \\
\text { (PE/DA; } \\
\text { EP/CP; PP/O) }\end{array}$ & 3840.3 & 557 & 6.9 & .114 & .688 & .708 & .633 & $\begin{array}{c}.084 \\
(.082-.087)\end{array}$ & 4.80 \\
\hline $\begin{array}{l}\text { Modelo 6F } \\
(27 \text { itens) }\end{array}$ & 1071.5 & 309 & 3.5 & .058 & .903 & .915 & .779 & $\begin{array}{c}.054 \\
(.051-.058)\end{array}$ & 1.46 \\
\hline
\end{tabular}

Nota. $\chi^{2}=$ Qui-quadrado; gl=Graus de liberdade; RMSEA (Root Mean Square Error of Approximation) e respetivo intervalo de confiança a 95\%; SRMR (Standardized Root Mean Square Residual); TLI (Tucker-Lewis Index); CFI (Comparative Fit Index); PNFI (Parsimonious Normed Fit Index); ECVI (Expected Cross-Validation Index).

restantes, apesar de este ajustamento não poder ser considerado satisfatório.

Não se tendo confirmado nenhuma das estruturas fatoriais analisadas, optou-se por refinar o modelo que mostrou melhor ajustamento. A reespecificação do modelo $6 \mathrm{~F}$ teve em consideração a magnitude das saturações fatoriais e a eventual presença de saturações duplas.

Todos os coeficientes de saturação estimados neste modelo foram estatisticamente significativos $(p<.001)$ e de magnitude moderada ou forte (os coeficientes padronizados oscilam entre $.403 \mathrm{e}$ .854 , respetivamente a ligação do item 6 ao fator $\mathrm{PP}$ e a ligação do item 31 ao fator $\mathrm{O}$ ). No entanto, os Índices de Modificação (MI) calculados pelo AMOS sugerem a existência de saturações duplas, indicando, por exemplo, que o item 26, originalmente integrado no fator $\mathrm{EP}$, se associa também ao fator PP (MI=54.0) e que o item 16, originalmente associado ao fator $\mathrm{PP}$, se liga ao fator DA (MI=51.0). São sugeridas ainda ligações entre itens associados ao mesmo fator (e.g., a ligação entre os itens 13 e 18 do fator CM, $\mathrm{MI}=33.1)$ e entre itens associados a fatores diferentes (e.g., a ligação entre o item 26 do fator EP e o item 19 do fator PP, MI=49.8), bem como correlação entre erros (e.g., entre os erros associados aos itens 9 e 10, MI=57.5). Apesar do valor elevado destes Índices de Modificação e da sua significância estatística, o facto de libertarmos o valor do parâmetro a eles associado não permite ainda assegurar o ajustamento do modelo $6 \mathrm{~F}$. Por outro lado, introduzir neste modelo pequenas alterações, geralmente consideradas aceitáveis na literatura quando se trata da estrutura fatorial de instrumentos psicométricos, tais como permitir a correlação entre os erros, não permitiu alcançar níveis de ajustamento satisfatórios. Assim, perante a diversidade de situações potencialmente problemáticas identificadas pelos Índices de Modificação e tendo como objetivo preservar uma estrutura fatorial simples (sem saturações duplas e com erros específicos não correlacionados), optou-se por eliminar os itens que contribuíam menos para os fatores respetivos e que se associavam de forma significativas a outros fatores, evitando desta forma saturações duplas. Este procedimento foi repetido até serem obtidos níveis satisfatórios de ajustamento, tendo sido necessário eliminar oito itens. À exceção dos indicadores diretamente associados à estatística $\chi^{2}$, todos os outros índices de ajustamento sugerem que o modelo $6 \mathrm{~F}$ assim revisto se ajusta à matriz de covariâncias determinada pelas respostas dos participantes à FMPS.

No Quadro 3 são apresentados os coeficientes padronizados de saturação para os 27 itens da versão revista da FMPS e os respetivos indicadores de fiabilidade individual $\left(R^{2}\right.$, correspondente à fração de variabilidade total do 
item explicada pelo fator que lhe está associado). $\mathrm{O}$ valor dos coeficientes de saturação é moderado ou forte, sendo sistematicamente mais elevado nos fatores Organização $(\mathrm{O})$ e Expetativas parentais (EP). Apenas dois itens (item 3 e item 17) têm coeficientes de saturação inferior a .5 , indicando baixa fiabilidade individual (mais de $75 \%$ da variância nas respostas a estes itens parece não depender do fator latente que lhes está associado). No total, um terço dos itens da escala apresenta indicadores de fiabilidade individual elevados (superiores a .6, tal como recomendado por alguns autores (Fornell \& Larcker, 1981).

Quadro 3. Estrutura Fatorial da FMPS Revista:

Coeficientes de Saturação Fatorial (Padronizados) e Indicadores Individuais de Fiabilidade $\left(\mathrm{R}^{2}\right)$

\begin{tabular}{|c|c|c|c|c|c|c|c|}
\hline Item & $\mathrm{PE}$ & PP & EP & $\mathrm{CP}$ & DA & $\mathrm{O}$ & $R^{2}$ \\
\hline 25 & .701 & & & & & & .492 \\
\hline 21 & .675 & & & & & & .455 \\
\hline 23 & .671 & & & & & & .450 \\
\hline 34 & .631 & & & & & & .398 \\
\hline 13 & .504 & & & & & & .254 \\
\hline 19 & & .780 & & & & & .608 \\
\hline 12 & & .685 & & & & & .469 \\
\hline 6 & & .574 & & & & & .330 \\
\hline 30 & & .562 & & & & & .316 \\
\hline 20 & & & .779 & & & & .607 \\
\hline 11 & & & .774 & & & & .600 \\
\hline 15 & & & .634 & & & & .402 \\
\hline 1 & & & .629 & & & & .395 \\
\hline 22 & & & & .765 & & & .585 \\
\hline 35 & & & & .658 & & & .434 \\
\hline 5 & & & & .555 & & & .308 \\
\hline 3 & & & & .477 & & & .228 \\
\hline 33 & & & & & .666 & & .444 \\
\hline 32 & & & & & .661 & & .437 \\
\hline 28 & & & & & .538 & & .289 \\
\hline 17 & & & & & .473 & & .224 \\
\hline 2 & & & & & & .800 & 639 \\
\hline 7 & & & & & & .824 & .679 \\
\hline 8 & & & & & & .796 & .634 \\
\hline 27 & & & & & & .808 & .653 \\
\hline 29 & & & & & & .849 & .720 \\
\hline 31 & & & & & & .855 & .730 \\
\hline
\end{tabular}

Nota. $\quad N=832 . \quad$ PE=Preocupação com os Erros; $\mathrm{PP}=$ Padrões Pessoais; $\mathrm{EP}=$ Expetativas Parentais; $\mathrm{CP}=$ Críticas Parentais; $\mathrm{DA}=$ Dúvidas sobre as Ações; $\mathrm{O}=$ Organização.

Todos os coeficientes de saturação fatorial significativos para $p<.001$
Os itens da versão revista da FMPS organizam-se em seis subescalas que avaliam as seis dimensões de perfecionismo subjacentes às respostas dadas a esta escala. Considerou-se ainda uma medida global de perfecionismo, integrando a resposta a todos os itens, com exceção dos associados à escala Organização (O), tal como tem vindo a ser feito por outros autores (e.g., Amaral et al., 2013; Cox et al., 2002; Lee \& Park, 2011). O Quadro 4 apresenta os valores de correlação entre as medidas proporcionadas pelas seis subescalas da FMPS, e que refletem as correlações entre as seis dimensões do perfecionismo.

Tal como esperado, a dimensão Organização (O) destaca-se por apresentar correlações de baixa magnitude com as restantes. Todas as outras dimensões mostram valores de correlação moderados entre si. É de destacar a correlação elevada entre Críticas Parentais (CP) e Expetativas Parentais (EP) (próxima de .6), indicadora de proximidade concetual entre estas duas dimensões.

De uma forma geral, o padrão de correlações entre as seis subescalas da FMPS revista reflete as estruturas fatoriais que têm vindo a ser propostas para esta escala (nomeadamente, a integração de $\mathrm{CP}$ e EP num único fator ou a associação entre PE e DA).

\section{Análise da fiabilidade das medidas}

Para avaliar a fiabilidade das seis subescalas da FMPS, recorreu-se à análise da consistência interna (alfa de Cronbach) e à análise da estabilidade temporal (teste-reteste).

O Quadro 4 apresenta os valores de consistência interna das seis subescalas, que se mostraram bastante satisfatórios (superiores a .7), com exceção da subescala Dúvidas sobre as Ações (DA) (.663). O número reduzido de itens (quatro) que compõem esta subescala pode ter contribuído para o valor obtido. Os coeficientes alfa não aumentam quando eliminado qualquer um dos itens da escala, à exceção do item 13 cuja eliminação beneficiou muito ligeiramente a escala PE.

A estabilidade temporal da FMPS foi avaliada através do método teste-reteste. Os valores elevados de correlação de Pearson entre os dois momentos de avaliação $(p<.001)$ evidenciaram 
Quadro 4. Correlação entre as Pontuações nas Subescalas da FMPS Revista, Coeficiente Alfa de Cronbach, Coeficiente Alfa de Cronbach quando Item Eliminado e Coeficiente de Correlação Teste-Reteste

\begin{tabular}{cccccccc}
\hline FMPS & PE & PP & EP & CP & DA & O & FMPS tot \\
\hline PE & - & & & & & & \\
PP & $.297^{* * *}$ & - & & & & & \\
EP & $.381^{* * *}$ & $.350^{* * *}$ & - & & & & \\
CP & $.436^{* * *}$ & .062 & $.560^{* * *}$ & - & & & \\
DA & $.428^{* * *}$ & $.181^{* * *}$ & $.192^{* * *}$ & $.401^{* * *}$ & - & & \\
O & -.049 & $.297 * * *$ & .008 & $-.070^{*}$ & .049 & - & \\
FMPS tot & $.760^{* * *}$ & $.557^{* * *}$ & $.745^{* * *}$ & $.712^{* * *}$ & $.626^{* * *}$ & .066 & - \\
\hline$\alpha$ & .765 & .745 & .792 & .698 & .663 & .924 & .853 \\
$\alpha-$ item & $.702-.769$ & $.646-.724$ & $.697-.769$ & $.585-.694$ & $.549-.653$ & $.904-.914$ & $.842-.853$ \\
$r$ (teste- & $.689 * * *$ & $.774 * * *$ & $.742^{* * * *}$ & $.699^{* * *}$ & $.741 * * *$ & $.888^{* * * *}$ & $.799 * * *$ \\
reteste) & & & & & & & \\
\hline
\end{tabular}

Nota. $N=\overline{832}$. $\mathrm{PE}=$ Preocupação com os Erros; $\mathrm{PP}=$ Padrões Pessoais; $\mathrm{EP}=$ Expetativas Parentais; $\mathrm{CP}=$ Críticas Parentais; DA=Dúvidas sobre as Ações; $\mathrm{O}=$ Organização; FMPS tot=Valor total do perfecionismo avaliado pela FMPS; $\alpha=$ Alfa de Cronbach; $\alpha$-item=Intervalo de valores do alfa de Cronbach caso se elimine qualquer um dos itens da escala; $r$ (testereteste $)=$ Coeficiente de correlação teste-reteste.

$* p<.05 ; * * p<.01 ; * * * p<.001$

boa estabilidade temporal da escala, com valores de correlação elevados. A correlação teste-reteste foi de .799 para a escala FMPS total, e oscilou entre .689 (PE) e $.888(\mathrm{O})$ para as subescalas (Quadro 4). No mesmo sentido, a análise dos níveis médios de resposta permitiu constatar que não existem diferenças significativas entre os dois momentos de avaliação, exceto na subescala Críticas Parentais (CP), onde, apesar de existir consistência nas respostas ( $r=.699)$, se observa um aumento significativo na pontuação entre os dois momentos $\quad\left(t_{(118)}=-2.5, \quad p=.014\right)$, embora de reduzida magnitude ( $d$ de Cohen=.12).

\section{Validade convergente e discriminante}

Considera-se que uma medida compósita apresenta validade convergente quando uma fração elevada da variância dos itens é atribuível unicamente ao fator que lhes está subjacente e não a outro. O índice VEM (Variância extraída média) avalia o grau em que a variância dos itens que refletem um fator é explicada por esse fator e deve assumir valor superior a .5 para que se possa considerar que a subescala utilizada é uma medida válida do fator (Fornell \& Larcker, 1981). Os resultados da análise da validade convergente da FMPS não são totalmente favoráveis: enquanto duas subescalas refletem o critério acima apontado (Organização: VEM=.675; Expetativas Parentais: VEM=.501), as restantes apontam índices de validade convergente inferiores ao desejável (Preocupação com os Erros: VEM=.410; Padrões Pessoais: VEM=.431; Críticas Parentais: VEM=.388; Dúvidas sobre Ações: VEM=.348). Estes resultados indicam que os itens que formam as diferentes subescalas de FMPS não propiciam medidas suficientemente puras dos construtos que a escala realmente pretende medir, o que poderá parcialmente resultar da correlação existente entre subescalas (Quadro 4).

Por sua vez, a validade discriminante avalia o grau em que os itens associados a um fator refletem preferencialmente esse fator e não outro. Para haver discriminação entre dois fatores, é necessário que a variância extraída média de cada fator seja superior à variância partilhada entre eles (ou seja, ao quadrado da correlação entre os fatores) (Fornell \& Larcker, 1981). No presente caso, as correlações estimadas entre os seis fatores são suficientemente baixas, para garantir que a percentagem de variância partilhada entre fatores seja claramente inferior ao grau em que cada fator é responsável pela variância dos seus respetivos itens. Apenas em dois fatores a validade discriminante está ameaçada: os fatores EP e CP partilham uma fração de variância relativamente elevada $\left(r^{2}=.441\right)$, valor superior à VEM associada a CP. Desta forma, mais uma vez, podemos ponderar que a FMPS não permite uma discriminação suficientemente clara entre as dimensões CP e EP. 
Quadro 5. Correlação entre as Subescalas da FMPS Revista e as Subescalas da HMPS

\begin{tabular}{cccccccc}
\hline \multicolumn{7}{c}{ FMPS } \\
\hline HMPS & PE & PP & EP & CP & DA & O & FMPS total \\
\hline PAO & $.302 * * *$ & $.700^{* * *}$ & $.334 * * *$ & .035 & $.148^{* * *}$ & $.345^{* * *}$ & $.452 * * *$ \\
PSP & $.618^{* * *}$ & $.307 * * *$ & $.508^{* * *}$ & $.543 * * *$ & $.439 * * *$ & -.043 & $.712 * * *$ \\
POO & -.044 & $.165 * * *$ & .043 & $-.115 * *$ & -.042 & $.113 * * *$ & .004 \\
HMPS total & $.441^{* * *}$ & $.637 * * *$ & $.458^{* * *}$ & $.214 * * *$ & $.255^{* * *}$ & $.245^{* * *}$ & $.596 * * *$ \\
\hline
\end{tabular}

Nota. $N=832$. HMPS=Escala Multidimensional de Perfecionismo de Hewitt e Flett (1991); PAO=Perfecionismo Autoorientado; PSP=Perfecionismo Socialmente Prescrito; POO=Perfecionismo Orientado para os Outros; HMPS total=Valor total de perfecionismo avaliado pela HMPS; FMPS=Escala Multidimensional de Perfecionismo de Frost et al. (1990); PP=Padrões Pessoais; $\mathrm{PE}=\mathrm{Preocupação} \mathrm{com} \mathrm{os} \mathrm{Erros;} \mathrm{EP}=$ Expetativas Parentais; $\mathrm{CP}=\mathrm{Cr}$ tícas Parentais; DA=Dúvidas sobre as Ações; O=Organização; FMPS total=Valor total do perfecionismo avaliado pela FMPS.

$* p<.05 ; * * p<.01 ; * * * p<.001$

\section{Validade concorrente}

Para o estudo da validade concorrente das subescalas da FMPS foram analisadas as relações entre essas medidas e as proporcionadas pela Escala Multidimensional de Perfecionismo HMPS (Hewitt \& Flett, 1991).

O Quadro 5 apresenta os coeficientes de correlação de Pearson entre as três subescalas da HMPS e as seis subescalas da versão revista da FMPS. As subescalas destes dois instrumentos mostram-se parcialmente correlacionadas. Observam-se correlações positivas moderadas e fortes entre as dimensões Perfecionismo Autoorientado (PAO) e Padrões Pessoais (PP) e entre a dimensão Perfecionismo Socialmente Prescrito (PSP) e as dimensões Preocupação com os Erros (PE), Expetativas Parentais (EP), Críticas Parentais (CP) e Dúvidas sobre as Ações (DA). As pontuações totais das duas escalas apresentam uma correlação também elevada ( $r=.596)$, sendo de destacar a correlação entre a subescala PSP e a pontuação total da FMPS $(r=.712)$ e entre a subescala PP e a pontuação total da HMPS $(r=.637)$. A subescala Perfecionismo Orientado para os Outros (POO) é aquela que apresenta um menor número de correlações significativas com as subescalas da FMPS.

\section{Diferença entre grupos}

A comparação entre grupos não mostrou diferenças estatisticamente significativas entre sexos $(d$ de Cohen <.1; $p>.1)$ nas diferentes medidas proporcionadas pela FMPS, exceto a presença de uma diferença moderada na subescala Organização (O) ( $d$ de Cohen $=.48 ; t_{(830)}=6.7$, $p<.001$ ), apresentando as mulheres pontuações médias superiores aos homens.
Considerando a distribuição da amostra por dois grupos etários, jovens até aos 19 anos, inclusive $(n=499,60 \%)$ e jovens adultos $(n=333)$, foi possível verificar que não existem diferenças estatisticamente significativas atribuíveis à idade ( $d$ de Cohen $<.2 ; p>.2$ ), exceto a presença de uma diferença de magnitude reduzida na subescala Organização $(\mathrm{O}) \quad\left(d\right.$ de Cohen=.21; $t_{(830)}=3.1$, $p=.002$ ), apresentando o grupo mais velho pontuação média superior à do grupo de adolescentes. Relativamente ao nível de ensino, observam-se diferenças estatisticamente significativas de magnitude reduzida na Preocupação com os erros (PE) e nas Críticas parentais (CP) ( $d$ de Cohen=.14; $t_{(830)}=-2.1$, $p=.040$ e $t_{(830)}=-2.6, p=.011$, respetivamente), obtendo os estudantes do ensino secundário pontuações superiores aos estudantes do ensino universitário. Em sentido inverso, os universitários apresentaram pontuações médias significativamente mais elevadas na subescala Organização (O), contudo a magnitude dessa diferença foi reduzida $\left(d\right.$ de Cohen $=.17 ; t_{(830)}=2.9$, $p=.004)$.

\section{Discussão}

Considerando a diversidade de soluções fatoriais propostas nas diferentes adaptações da Escala Multidimensional de Perfecionismo de Frost et al. (1990), o principal objetivo deste estudo foi contribuir para a clarificação da dimensionalidade da FMPS recorrendo a uma abordagem confirmatória numa amostra alargada de jovens e jovens adultos portugueses. A análise dos vários modelos fatoriais apresentados na literatura permitiu considerar o modelo 
multidimensional de seis fatores aquele que melhor representa os dados recolhidos com a versão portuguesa da FMPS, replicando assim a estrutura original da escala.

Os índices de ajustamento sugeriram que o modelo de seis fatores era o que mais se aproximava dos níveis de qualidade comumente exigidos (Kline, 2011), sem que no entanto fosse garantido um ajustamento satisfatório. De modo a corresponder às exigências deste tipo de análise, e à semelhança de outros estudos de adaptação da escala, foram eliminados alguns itens que apresentavam saturações em mais do que um fator. Tal como aconteceu em algumas investigações que replicaram a estrutura original da FMPS (e.g., Parker \& Adkins, 1995; Rheaume et al., 1995), a eliminação de itens foi inevitável. A maioria dos estudos de adaptação não manteve os 35 itens da versão original independentemente da estrutura fatorial encontrada (seis, cinco, quatro ou três fatores). É de notar que a versão portuguesa de Amaral et al. (2013) foi uma das que manteve todos os itens, sugerindo os autores duas soluções fatoriais (seis e quatro dimensões).

A análise da consistência interna da versão revista de 27 itens apresentou coeficientes bastante satisfatórios, tanto para a pontuação total da escala, como para as diferentes subescalas, à exceção da subescala Dúvidas sobre as Ações (DA), onde os coeficientes de consistência interna foram ligeiramente inferiores ao mínimo recomendável (.7). Estes resultados parecem ser concordantes com o estudo original e com a grande maioria dos estudos de adaptação realizados noutros contextos e países (e.g., Cox et al., 2002; Lee \& Park, 2011; Parker \& Stumpf, 1995), onde sistematicamente esta dimensão mostra valores de consistência interna inferiores às restantes dimensões. A este propósito, numa meta-análise sobre a generalização da fiabilidade da FMPS, Ha, Lee e Puig (2010) concluíram que a subescala DA é aquela que apresenta valores médios de fiabilidade mais baixos em todos os estudos analisados. Originalmente esta é já uma subescala com um número reduzido de itens (quatro), inibindo a eliminação dos itens com menor consistência, que poderia aumentar a fiabilidade da subescala (Cortina, 1993).

Verificou-se uma boa estabilidade temporal das medidas num intervalo de tempo de Revista Iberoamericana de Diagnóstico y Evaluación - e Avaliação Psicológica. RIDEP · Nº44 · Vol.2 · 28-43 2017 aproximadamente um mês. De acordo com Gelabert et al. (2011), este resultado poderá sustentar a noção de que este instrumento avalia o perfecionismo como um traço de personalidade.

A análise da validade convergente não é totalmente satisfatória, indiciando que alguns dos itens que compõem as diferentes subescalas da FMPS não refletem claramente os construtos que a escala pretende medir. Os itens das subescalas Organização (O) e Expetativas Parentais (EP) permanecem bastante nítidos, contudo, alguns itens das subescalas Preocupação com os Erros (PE), Padrões Pessoais (PP), Dúvidas sobre as Ações (DA) e Críticas Parentais (CP) não apresentam saturações fatoriais satisfatórias. A análise da validade discriminante está garantida, à exceção de dois fatores, CP e EP, que partilham uma variância relativamente elevada, não possibilitando desta forma uma discriminação suficientemente clara entre estas dimensões. Este resultado vai ao encontro da problematização de alguns autores quanto à distinção entre a perceção de pais com elevadas expetativas e de pais demasiado críticos, uma vez que nem sempre os filhos percecionam expetativas elevadas e críticas parentais como duas atitudes diferentes (Cheng et al., 1999). Além disso, não podemos deixar de sublinhar que a solução de cinco fatores mais comum mostra sistematicamente índices adequados de ajustamento nos modelos que agrupam estas duas dimensões (Críticas e Expetativas Parentais) numa única (e.g., Cox et al., 2002; Lee \& Park, 2011), sugerindo a tendência não discriminatória das dimensões que avaliam a componente desenvolvimental do perfecionismo.

No que se refere à validade concorrente, a FMPS associou-se no sentido esperado a uma medida multidimensional de avaliação do perfecionismo (HMPS). Os resultados entre as subescalas destas medidas foram consistentes com os resultados de estudos prévios (e.g., Frost et al., 1993; Hewitt \& Flett, 2004), apresentando uma forte relação entre as dimensões da componente intrapessoal (Perfecionismo Auto-Orientado, Padrões Pessoais e Organização) e as dimensões da componente interpessoal (Perfecionismo Socialmente Prescrito, Expetativas Parentais, Críticas Parentais e Dúvidas sobre as Ações). 
Os participantes do sexo feminino mostraram pontuações mais elevadas na dimensão Organização do que os participantes do sexo masculino. Este resultado parece ser transversal à maioria dos estudos com amostras de estudantes de ambos os sexos (Egan et al., 2011). Culturalmente, a ordem e a organização parecem ser características estimuladas e espectáveis mais nas mulheres do que nos homens. Também o efeito da idade se manifestou unicamente por um nível médio de Organização mais elevado entre os participantes mais velhos, resultado que poderá justificar por que razão os estudantes universitários apresentaram valores superiores de Organização comparativamente aos estudantes mais jovens. Por outro lado, os estudantes do ensino secundário apresentaram níveis de Preocupação com os Erros e de Críticas Parentais significativamente mais elevados. Se pensarmos que a grande maioria dos estudantes do secundário que participou neste estudo frequentava os últimos anos deste ciclo de estudos, e sendo esta uma fase exigente e decisiva, o medo de falhar, a preocupação com os erros e a maior proximidade com os pais poderão á ter contribuído para esta diferença nos resultados.

Podemos apontar como principais limitações o facto de a amostra ser exclusivamente composta por jovens estudantes a frequentar uma instituição do ensino secundário ou universitário da região do Algarve, o que dificulta a generalização direta dos resultados a diferentes populações e contextos.

Contudo, apesar desta limitação e da necessidade de eliminar alguns itens para obter índices de ajustamento satisfatórios ao modelo de seis dimensões, os resultados permitem concluir que esta versão portuguesa revista da FMPS apresenta uma boa fiabilidade e uma adequada validade, constituindo-se como um instrumento fiável e validado para o estudo do perfecionismo em Portugal.

\section{Referências}

Adler, A. (1956). The neurotic disposition. In H. L. Ansbacher \& R. R. Ansbacher (Eds.), The Individual Psychology of Alfred Adler. New York: Harper \& Row.
Altstotter-Gleich, C., \& Bergemann, N. (2006). The statistics and validity of German version of a Multidimensional Perfectionism Scale of Frost, Marten, Lahart and Rosenblate (MPSF). Diagnostica, 52(3), 105-118. doi:10.1026/0012-1924.52.3.105

Amaral, A., Soares, M., Pereira, A., Bos, S., Marques, M., Valente, J., ... Macedo, A. (2013). Frost Multidimensional Perfectionism Scale: The Portuguese version. Revista de Psiquiatria Clínica, 40(4), 144-149.

Azevedo, M. H., Bos, S. C., Soares, M. J., Marques, M., Pereira, A. T., Maia, B., ... Macedo, A. (2010). Longitudinal study on perfectionism and sleep disturbance. The World Journal of Biological Psychiatry, 11(2), 476-485.

Blatt, S. J., D'Afflitti, J. P., \& Quinlan, D. M. (1976). Experiences of depression in normal young adults. Journal of Abnormal Psychology, 85, 383-389.

Burns, D. D. (1980). The perfectionists script for self-defeat. Psychology Today, 14(6), 34-52.

Carvalho, R. G., \& Novo, F. R. (2014). Dimensões da personalidade e comportamentos de risco na adolescência: Um estudo com a versão portuguesa do MMPI-A. Revista Iberoamericana de Diagnóstico y Evaluación - e Avaliação Psicológica, 1(37), 203-222.

Chan, D. W. (2009). Dimensionality and typology of perfectionism: The use of the Frost Multidimensional Perfectionism Scale with chinese gifted students in Hong Kong. Gifted Child Quarterly, 53(3), 174-187. doi:10.1177/0016986209334963

Cheng, S. K., Chong, G. H., \& Wong, C. W. (1999). Chinese Frost Multidimensional Perfectionism Scale: A validation and prediction of self-esteem and psychological distress. Journal of Clinical Psychology, 55(9), 1051-1061.

Cohen, J. (1988). Statistical power analysis for the behavioural sciences $\left(2^{\text {ed }}\right)$. New York: Academic Press.

Cortina, J. M. (1993). What is coefficient alpha? An examination of theory and applications. Journal of Applied Psychology, 78, 98-104.

Cox, B. J., Enns, M. W., \& Clara, I. P. (2002). The multidimensional structure of perfectionism in clinically distressed and V Vol.2 $28-43 \cdot 2017$ 
college student samples. Psychological Assessment, 14(3), 365-373. doi: 10.1037//1040-3590.14.3.365

Egan, S. J., Wade, T. D., \& Shafran, R. (2011). Perfectionism as a transdiagnostic process: A clinical review. Clinical Psychology Review, 31, 203-212.

Flett, G. L., Blankstein, K. R., Hewitt, P. L., \& Koledin, S. (1992). Components of perfectionism and procrastination in college students. Social Behavior and Personality, 20(2), 85-94.

Flett, G. L., Hewitt, P. L., Oliver, J. M., \& Macdonald, S. (2002). Perfectionism in children and their parents: A developmental analysis. In G. L. Flett \& P. L. Hewitt (Eds.), Perfectionism: Theory, research, and treatment (pp. 89-132). Washington, D.C.: American Psychological Association.

Fornell, C., \& Larcker, D. F. (1981). Evaluating structural equation modelling with unobserved variables and measurement error. Journal of Marketing Research, 18(1), 39-50.

Frost, R. O., Heimberg, R. G., Holt, C. S., Mattia, J. I., \& Neubauer, A. L. (1993). A comparison of two measures of perfectionism. Personality and Individual Differences, 14(1), 119-126.

Frost, R. O., Marten, P., Lahart, C., \& Rosenblate, R. (1990). The dimensions of perfectionism. Cognitive Therapy and Research, 14(5), 449468.

Garner, D. M., Olmsted, M. P., \& Polivy, J. (1983). Development and validation of a multidimensional eating disorder inventory for anorexia nervosa and bulimia. International Journal of Eating Disorders, 2, 15-34.

Gautreau, C., Sherry, S., Mushquash, A., \& Stewart, S. (2015). Is self-critical perfectionism an antecedent of or a consequence of social anxiety, or both? A 12month, three-wave longitudinal study. Personality and Individual Differences, 82, 125-130. doi:10.1016/j.paid.2015.03.005

Gelabert, E., García-Esteve, L., Martín-Santos, R., Gutiérrez, F., Torres, A., \& Subirà, S. (2011). Psychometric properties of the spanish version of the Frost Multidimensional Perfectionism Scale in women. Psicothema, 23(1), 133-139.
Ha, J. H., Lee, S. M., \& Puig, A. (2010). A reliability generalization study of the Frost Multidimensional Perfectionism Scale (FMPS). Psychological Reports, 107(1), 95112. doi:10.2466/03.09.20.PR0.107.4.95-112

Hamachek, D. E. (1978). Psychodynamics of normal and neurotic perfectionism. Psychology: A Journal of Human Behavior, 15(1), 27-33.

Harvey, B., Pallant, J., \& Harvey, D. (2004). An evaluation of the factor structure of the Frost Multidimensional Perfectionism Scale. Educational and Psychological Measurement, 64(6), 1007-1018. doi:10.1177/001316440426 4842

Hawkins, C. C., Watt, H. M. G., \& Sinclair, K. E. (2006). Psychometric properties of the Frost Multidimensional Perfectionism Scale with australian adolescent girls - Clarification of multidimensionality and perfectionist typology. Educational and Psychological Measurement, 66(6), 1001-1022. doi:10.1177/0013164405285909

Hewitt, P. L., \& Flett, G. L. (1991). Perfectionism in the self and social context: Conceptualization, assessment, and association with psychopathology. Journal of Personality and Social Psychology, 60(3), 456-470.

Hewitt, P. L., \& Flett, G. L. (2004). Multidimensional Perfectionism Scale (MPS): Technical manual. Toronto: Multi-Health Systems.

Hewitt, P. L., Flett, G. L., Sherry, S. B., \& McGee, B. (2003). Perfectionism is Multidimensional: A reply to Shafran, Cooper and Fairburn (2002). Behaviour Research and Therapy, 41, 1221-1236.

Hodgson, R. J., \& Rachman, S. (1977). Obsessional-compulsive complaints. Behaviour Research and Therapy, 15(5), 389395.

Hollender, M. H. (1965). Perfectionism. Comprehensive Psychiatry, 6(2), 94-103.

Horney, K. (1950). Neurosis and Human Growth. New York: Norton.

Kagan, M. (2011). Psychometric properties of the Turkish version of the Frost Multidimensional Perfectionism Scale. Anatolian Journal of Psychiatry, 12(3), 192-197. 
Kline, R. B. (2011). Principles and Practice of Structural Equation Modeling ( $3^{\text {rd }}$ ed.). New York: The Guilford Press.

Lee, D. G., \& Park, H. J. (2011). Cross-cultural validity of the Frost Multidimensional Perfectionism Scale in Korea. Counseling Psychologist, $\quad 39(2), \quad 320-345$. doi: $10.1177 / 0011000010365910$

Macedo, A., Soares, M., Amaral, A., Nogueira, V., Madeira, N., Roque, C., ... Pereira, A. (2015). Repetitive negative thinking mediates the association between perfectionism and psychological distress. Personality and Individual Differences, 72, 220-224. doi:10.1016/j.paid.2014.08.024

Missildine, W. H. (1963). Your Inner Child of the Past. New York.

Moreira, J. M. (2009). Questionários: Teoria e Prática. Coimbra: Almedina.

O'Connor, R. C. (2007). The relations between perfectionism and suicidality: A systematic review. Suicide and Life-Threatening Behavior, 37(6), 698-714.

Oros, L. B. (2003). Medición del perfeccionismo infantil: Desarrollo y validación de una escala para niños de 8 a 13 años de edad. Revista Iberoamericana de Diagnóstico y Evaluación - e Avaliação Psicológica, 15(2), 99-112.

Pacht, A. R. (1984). Reflections on Perfection. American Psychologist, 39(4), 386-390.

Paredes, K. F., Mancilla-Diaz, J. M., Rayon, G. A., Arevalo, R. V., \& Aguilar, X. L. (2010). Factor structure and internal consistency of the Perfectionism Multidimensional Scale. Revista Mexicana De Psicologia, 27(2), 143149.

Parker, W. D., \& Adkins, K. K. (1995). A psychometric examination of the Multidimensional Perfectionism Scale. Journal of Psychopathology and Behavioral Assessment, 17(4), 323-334.

Parker, W. D., \& Stumpf, H. (1995). An examination of the Multidimensional Perfectionism Scale with a sample of academically talented children. Journal of Psychoeducational Assessment, 13(4), 372383.

Pinto, A., Greene, A., Storch, E., \& Simpson, H. (2015). Prevalence of childhood obsessivecompulsive personality traits in adults with obsessive compulsive disorder versus obsessive compulsive personality disorder. Journal of Obsessive-Compulsive and Related Disorders, 4, 25-29. doi:10.1016/j.jocrd.2014. 11.002.

Purdon, C., Antony, M. M., \& Swinson, R. P. (1999). Psychometric properties of the Frost Multidimensional Perfectionism Scale in a clinical anxiety disorders sample. Journal of Clinical Psychology, 55(10), 1271-1286.

Rheaume, J., Freeston, M. H., Dugas, M. J., Letarte, H., \& Ladouceur, R. (1995). Perfectionism, responsibility and obsessivecompulsive symptoms. Behaviour Research and Therapy, 33(7), 785-794.

Shafran, R., Cooper, Z., \& Fairburn, C. G. (2002). Clinical perfectionism: A cognitivebehavioural analysis. Behaviour Research and Therapy, 40(7), 773-791.

Shafran, R., Cooper, Z., \& Fairburn, C. G. (2003). "Clinical perfectionism" is not "multidimensional perfectionism": A reply to Hewitt, Flett, Besser, Sherry, \& McGee. Behaviour Research and Therapy, 41(10), 1217-1220.

Slaney, R. B., Rice, K. G., Mobley, M., Trippi, J., \& Ashby, J. S. (2001). The revised Almost Perfect Scale. Measurement and Evaluation in Counseling and Development, 34(3), 130-145.

Soares, M. J., Gomes, A. A., Macedo, A. F., \& Azevedo, M. H. P. (2003). Escala multidimensional de perfeccionismo: Adaptação à população portuguesa. Revista Portuguesa de Psicossomática, 5(1), 46-55.

Stallman, H., \& Hurst, C. (2011). The factor structure of the Frost Multidimensional Perfectionism Scale in university students. Australian Psychologist, 46(4), 229-236. doi:10.1111/j.1742-9544.2010.00010.x

Stoeber, J. (1998). The Frost Multidimensional Perfectionism scale revisited: More perfect with four (instead of six) dimensions. Personality and Individual Differences, 24(4), 481-491.

Stumpf, H., \& Parker, W. D. (2000). A hierarchical structural analysis of perfectionism and its relation to other personality characteristics. Personality and Individual Differences, 28(5), 837-852. 
van de Vijver, F. J. R., \& Hambleton, R. K. (1996). Translation tests: Some practical guidelines. European Psychologist, 1, 89-99.

Zi, F., \& Zhou, X. (2004). Chinese Frost's Multidimensional Perfectionism Scale: A validation and prediction of depression, anxiety, and shyness. International Journal of Psychology, 39(5-6), 550-550. 